\title{
LE DOSAGE DES ACIDES GRAS FIXES ET DES SUBSTANCES INSAPONIFIABLES DES TISSUS VÉGÉTAUX. ÉTUDE DE LA COMPOSITION DE QUELQUES ALIMENTS DES ANIMAUX
}

PAR

\author{
A. M. LEROY et A. FRANÇOIS (1)
}

Laboratoire de recherches de Zootechnie de l'Institut national agronomique

On désigne généralement sous le nom de "Matières Grasses " les substance, qui sont extraites par certains solvants organiques (en particulier l'éther sulfurique, l'éther de pétrole, le benzène), dans un appareil déterminé (de Soxhlet ou de Kumagawa, par exemple), la température du solvant et la durée de l'extraction étant précisées.

Déjà, dès rgo8, INABA (4) avait signalé que le traitement des tissus végétaux par l'éther absolu dans un appareil de Soxhlet n'extrait pas la totalité des lipides. Il indiquait que ce solvant peut, en revanche, entraîner des quantités importantes d'impuretés. D'autres auteurs ont récemment insisté sur l'entraînement de certaines substances azotées telles que l'urée par l'éther (I2). Néanmoins, la méthode de dosage des matières grasses par extraction directe présente de tels avantages de commodité qu'elle est encore utilisée très fréquemment. Elle est, par exemple, la méthode officielle préconisée par 1'A. O. A. C. (I) pour les aliments des animaux.

La technique de Kumagawa et Suto (5), modifiée par LEmeland (7) permet d'extraire quantitativement les acides gras et l'insaponifiable des tissus animaux. TERroINe, LEPAGE, Véchor et WolfF (I4) ont appliqué cette méthode aux tissus végétaux et l'ont comparée aux résultats obtenus en substituant, à l'extraction alcoolique, qui constitue le premier temps du dosage, une hydrolyse en milieu acide destinée à dissoudre partiellement la substance. Ces auteurs ont montré que le poids d'extrait brut, obtenu par traitement prolongé au soxhlet par l'éther éthylique ou l'éther de pétrole, est très voisin de celui de la somme "glycérides + insaponifiable " dosé par la méthode de KumaGAWA, lorsqu'il s'agit de substances renfermant des quantités très élevées de graisses. Il s'en éloigne considérablement, au contraire, dans le cas de matières

(1) Avec la collaboration de Mile Sknnedot. 
pauvres en corps gras. Ils concluent que la méthode de simple extraction de Soxhlet est à rejeter dans le cas de ces dernières.

MAYNARD et ses collaborateurs (9) utilisent également la méthode de KUMAGAWA, mais ne séparent pas les substances insaponifiables, qu'ils dosent avec les acides gras.

Plusieurs auteurs (I5) (II) (I2) (2) ont confirmé récemment que l'extraction par l'éther ne permet pas d'obtenir la totalité des acides gras des fourrages et que la méthode d'hydrolyse acide conduit à des résultats plus élevés.

La méthode généralement utilisée en France pour la détermination des matières grasses dans les aliments des animaux comporte une extraction préalable à l'alcool, puis une extraction à l'éther (6). Le premier traitement permet de détruire les cenapses lipo-protéiques et d'extraire une partie des lipides. Dès I9I9, BIFDERMANN émettait, en effet, l'opinion que les composés lipo-protéiques des cellules végétales sont très stables et persistent après la mort. Un traitement par l'alcool bouillant, puis par l'éther ou par le chloroforme permet d'extraire les lipides (voir MACHEBCEUf) (8).

Nous possédons peu de données sur la teneur en acides gras et en substances insaponifiables des principaux aliments des animaux. Le présent travai1 a pour but de combler partiellement cette lacune. Nous avons voulu, en outre, comparer le taux de matières grasses dosé par extraction directe à 1'alcool et à l'éther à la somme glycérides + insaponifiable dosés après hydrolyse et saponification.

\section{DOSAGE DES SUBSTANCES INSAPONIFIABLES ET DES ACIDES GRAS FIXES TOTAUX}

\section{Aliments étudiés}

Nos essais ont porté sur un certain nombre d'aliments : orge, avoine, son de blé, tourteaux de lin et d'arachide, foin de prairie, foin de luzerne, betteraves, pulpe de betteraves. Ces fourrages constituent généralement la base de 1'alimentation des animaux. Or, en dehors de son aspect énergétique, le problème de l'apport de matières grasses alimentaires peut revêtir une certaine importance, en particulier pour les femelles laitières. En outre, des dosages ont été effectués sur un certain nombre de fèces de bovins. Les mesures de digestibilité des lipides chez ces animaux feront l'objet d'une publication ultérieure. Les fèces contiennent des lipides sous forme de savons dont il convient de libérer les acides gras par une hydrolyse prolongée. L'élimination des sels biliaires et des pigments biliaires est favorisée par cette hydrolyse. D'autre part, les corps bactériens contenus dans les fèces renferment des lipides qu'il est difficile d'extraire directement. 


\section{Choix de la technique analytique}

Nous avons d'abord tenté d'utiliser la technique proposée par 'TERroINE et ses collaborateurs. Nous nous sommes heurtés à certaines difficultés en ce qui concerne la séparation de la couche d'éther de pétrole. Il se forme souvent des émulsions et la méthode devient, alors, peu fidèle.

QUETEL (IO) signale également la formation d'émulsions et préconise un milieu alcoolique à $50 \%$. Nous avons constaté que cette méthode élimine les inconvénients dus à une mauvaise décantation et permet d'obtenir des résultats reproductibles. La technique décrite ci-après comprend donc l'hydrolyse acide, préconisée par TERROINE et ses collaborateurs et l'extraction des acides gras et des insaponifiables en milieu alcoolique à $50 \%$.

L'extraction des acides gras et des insaponifiables dans une ampoule à décantation ne permet pas de traiter une grande quantité de substance. Ce fait diminue la précision de la méthode, mais cette critique est surtout valable dans le cas des substances insaponifiables, dont le taux est toujours faible.

Dans une série d'essais préalables, nous avons vérifié que le cholestérol (pris comme insaponifiable-type) et l'acide stéarique sont quantitativement extraits par l'éther de pétrole en présence d'un milieu alcoolique à $50 \%$.

\section{Mode opératoire détaillé}

Traiter $2 \mathrm{~g}$ de substance pulvérisée finement (passage au tamis 40) par IOo cc d'acide chlorhydrique à Io $\%$ en volume (acide chlorhydrique $d=$ I, I9 90 cc-eau Io cc), pendant 6 heures, au bain-marie bouillant, sous réfrigérant à reflux. Neutraliser, au moyen d'une solution de potasse à $30 \%$ (vérifier le virage à la touche, en utilisant la phénolphtaléine comme indicateur). Ajouter Ioo cc d'alcool à $96^{\circ}$, puis $25 \mathrm{cc}$ d'une solution de potasse alcoolique à $50 \%$. Le milieu final possède une concentration en potasse d'environ $5 \%$ et un titre alcoolique voisin de 50 .

Saponifier pendant 2 heures à l'ébullition sous réfrigérant à reflux. Après refroidissement, extraire 1'insaponifiable par 80 , 70, 50 et 50 cc d'éther de pétrole (P. E. 50-60). Réunir les solutions d'insaponifiable dans une ampoule à décantation. Laver par $50 \mathrm{cc}$ d'une solution de potasse O. I. N. dans l'alcool à $50^{\circ}$. Réunir les liquides de lavage à la solution des acides gras. Neutraliser alors cette solution au moyen d'acide chlorhydrique au tiers (à la touche) en ajoutant l'acide doucement. Puis ajouter encore doucement ro à I 5 cc d'acide chlorhydrique pur $(d=$ I,I $)$. Noter le volume total d'acide chlorhydrique et ajouter un volume égal d'alcool à $9^{\circ}$ afin de maintenir la concentration alcoolique constante.

Extraire les acides gras par Ioo, Ioo puis $50 \mathrm{cc}$ d'éther de pétrole (P. E. $\left.50-60^{\circ}\right)$. Laver les extraits réunis par $50 \mathrm{cc}$ d'alcool à $50^{\circ}$. 
Evaporer les extraits d'insaponifiable et d'acides gras au bain-marie, sous vide, reprendre le résidu par quelques cc d'éther de pétrole. Filtrer sur un creuset de verre fritté $\mathbf{n}^{0}$ 2. Terminer la dessiccation sous vide, à la température ordinaire, dans un dessiccateur garni d'anhydride phosphorique. Placer ensuite le ballon à l'étuve $40^{\circ}$ jusqu'à poids constant.

(Une étuve à vide peut être avantageusement utilisée.)

\section{Dosage des matières grasses totales}

La technique de dosage est celle qui est généralement utilisée en France pour l'analyse des aliments des animaux. Elle comporte une extraction à l'alcool pendant 4 heures, puis une extraction à l'éther pendant 2 heures, dans un appareil de Soxhlet (6).

\section{Résultats obtenus}

Le tableau I réunit les valeurs obtenues.

Les résultats de deux dosage successifs d'acides gras(soit $a$ et $b$, par exemple) et d'insaponifiables, effectués sur un même produit, y sont indiqués, ainsi que la moyenne des deux déterminations $\frac{(a+b)}{2}$.

L'écart \% entre les deux déterminations est calculé de la façon suivante : $\frac{a-b}{\frac{a+b}{2}} \times$ roo. Cette valeur $b$ représente le double de l'erretur définie par l'écart entre chacune des valeurs et leur moyenne arithmétique.

Le taux de glycérides est obtenu en multipliant le taux d'acides gras par le facteur I,046 (d'après KumaGawA), ce qui revient à poser que les acides gras sont engagés dans des tryglycérides. L'écart entre la somme glycérides + insaponifiables et taux de matières grasses totales, est calculé en prenant pour base de référence la somme glycérides + insaponifiables. Un écart positif indique, par exemple, que le taux de matières grasses totales est supérieur à celui de la somme glycérides + insaponifiables.

Il convient de remarquer que les acides gras engagés dans des lipides complexes-phospholipides. en particulier, sont dosés avec les acides gras des glycérides.

Les acides gras volatils sont éliminés au cours du dosage et l'on n'obtient finalement que des acides à poids moléculaire élevé.

La fidélité de la méthode de dosage des acides gras est très satisfaisante. Sur 43 déterminations, 25 présentent un écart inférieur à $3 \%$ et 33 un écart inférieur à $5 \%$, entre deux dosages successifs sur un même échantillon.

Le dosage de l'insaponifiable présente une fidélité moins satisfaisante, en raison du faible taux de ces substances, qui entraîne une erreur relative plus importante. 


\section{Discussion des résultats}

Le tableau I montre que l'écart entre la somme "glycérides + insaponifiables " et "matières grasses totales " est relativement faible dans le cas des tourteaux oléagineux. Il semble, toutefois, que l'extraction à l'alcool et à l'éther entraîne, dans ce cas, une petite quantité de substances non lipidiques. Les céréales et le son de blé cèdent difficilement leurs lipides à l'alcool et à l'éther. Il existe un écart de l'ordre de Io à $15 \%$ entre les deux séries de valeurs. En revanche, dans le cas des foins et des fèces d'animaux nourris avec du foin, la quantité de substances extraites par l'alcool et à l'éther est plus élevée que la quantité d'acides gras et d'insaponifiables extraits après hydrolyse. Ce fait n'est pas surprenant, car on sait que la chlorophylle est entraînée par l'alcool et par 1'éther, alors qu'elle est éliminée sous forme d'acides chlorophylliquesinsolubles dans l'éther de pétrole (Chibnallet Chonnon - 3) dans la méthode par hydrolyse. Les résuitats les plus irréguliers sont obtenus dans le cas des betteraves, pour lesquelles l'écart entre les valeurs correspondant à chacune des méthodes peut être soit positif, soit négatif. Ceci semble résulter de la dessiccation des racines avant l'analyse. Les fragments du végétal subissent, au cours de la déshydratation à l'étuve, une modification physique un durcissement, qui rend l'extraction des lipides très irrégulière.

Ce fait met en évidence l'importance de l'état physique de la substance pour l'extraction des lipides. La finesse de mouture du produit joue vraisemblablement un rôle important. Il serait, sans doute, nécessaire de procéder à de oouveaux broyages de la substance et de poursuivre l'extraction pendant plusieurs heures encore.

L'étude de QUETEr a d'ailleurs mis en évidence l'influence de la durée de l'extraction sur le taux des lipides obtenus. Il semble qu'une extraction de 8 à 13 heures, dans un appareil de KumaGawa, soit nécessaire pour obtenir, par entraînement dans l'alcool bouillant, la presque totalité des lipides. Il semble que les durées d'extraction, actuellement utilisées, soient trop brèves et que le temps de 8 heures, indiqué par Kumagawa, soit indispensable.

Toutefois, l'analyse en série exige un procédé d'analyse rapide, précis et fidèle.

Guidé par ce triple objectif, il serait nécessaire d'étudier une technique“de dosage répondant à ces préoccupations.

Quoi qu'il en soit, la méthode de dosage des matières grasses au moyen d'un seul solvant tel que l'éther ou l'éther de pétrole est à rejeter à fortiori, puisqu'elle conduit à des résultats plus faibles que ceux obtenus au moyen de la double extraction à l'alcool et à l'éther. 


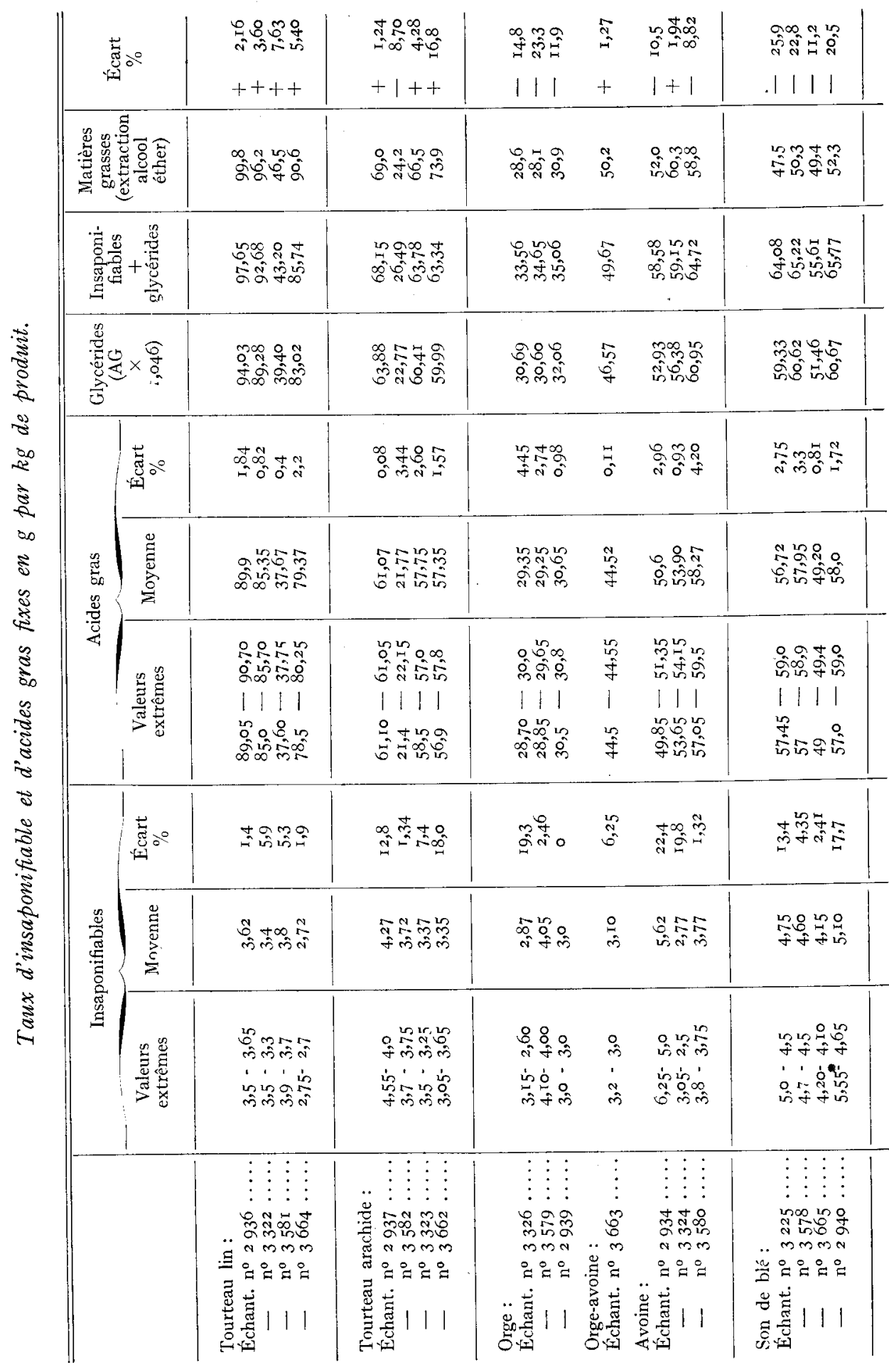




\begin{tabular}{|c|c|c|c|c|c|}
\hline 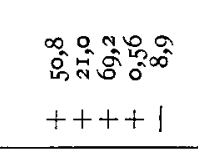 & 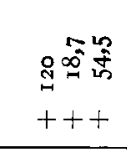 & 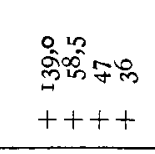 & 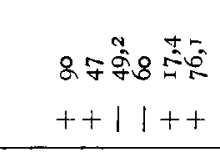 & $\stackrel{+}{+}$ & 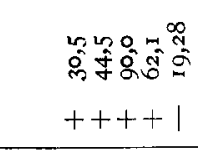 \\
\hline 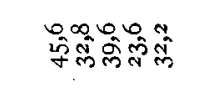 & 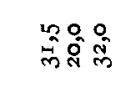 & 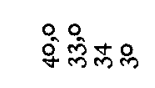 & 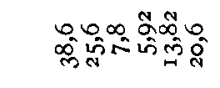 & $\stackrel{\circ}{\circ}$ & 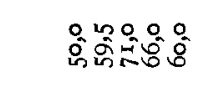 \\
\hline 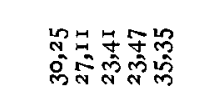 & 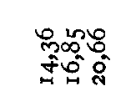 & 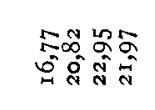 & 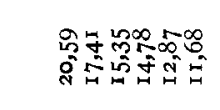 & $\frac{9}{9}$ & 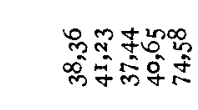 \\
\hline 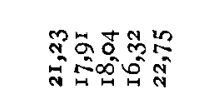 & 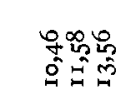 & 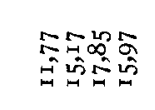 & 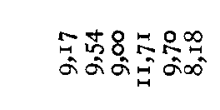 & $\stackrel{\infty}{s}$ & 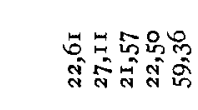 \\
\hline 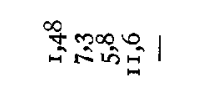 & 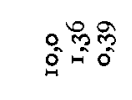 & 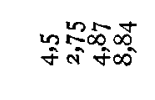 & 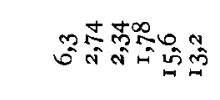 & $\dddot{2}$ & 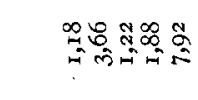 \\
\hline 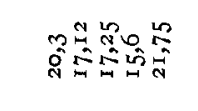 & 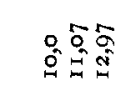 & 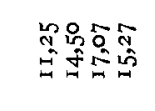 & $=$ & $\stackrel{\leftrightarrow}{\sharp}$ & 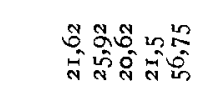 \\
\hline 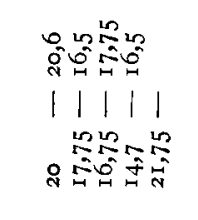 & 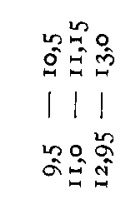 & 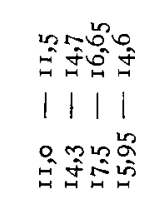 & 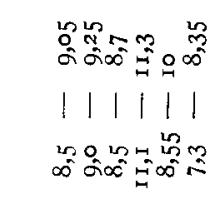 & 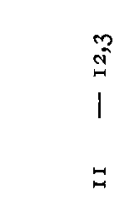 & 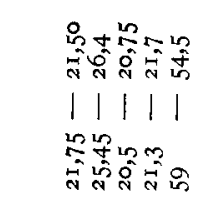 \\
\hline 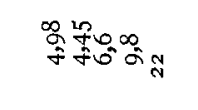 & 的员苛 & 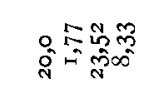 & 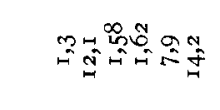 & $\overrightarrow{5}$ & 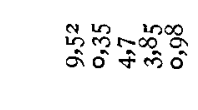 \\
\hline 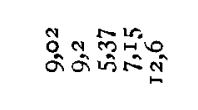 & 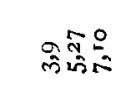 & 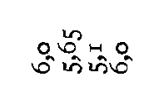 & 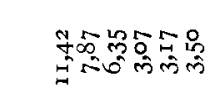 & $\stackrel{\infty}{\infty}$ & 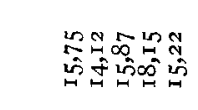 \\
\hline 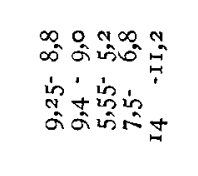 & 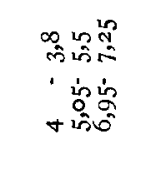 & 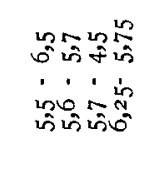 & 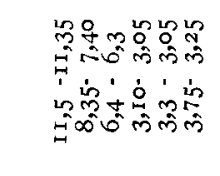 & $\stackrel{0}{\vdots}$ & 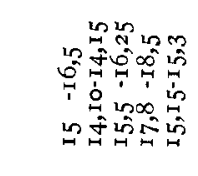 \\
\hline 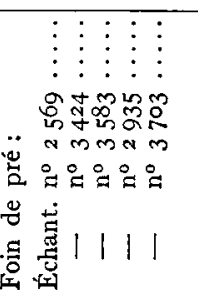 & 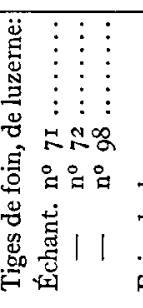 & $\begin{array}{l}0 \\
0 \\
0\end{array}$ & 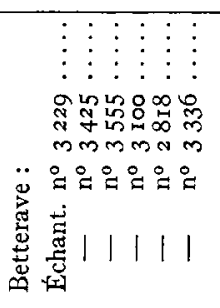 & 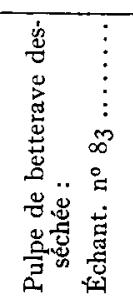 & 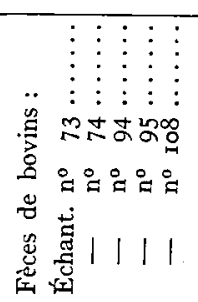 \\
\hline
\end{tabular}




\section{RESUME ET CONCLUSIONS}

I ${ }^{0}$ Les substances insaponifiables et les acides gras fixes des fourrages ont été dosés au moyen d'une méthode qui comporte les opérations suivantes; I0 Hydrolyse prolongée de la substance par l'acide chlorhydrique à Io \% ; $2^{\circ}$ Saponification en milieu alcoolique à $50 \% ; 3^{\circ}$ Extraction des insaponifiables par l'éther de pétrole 50-60; $4^{\circ}$ Libération des acides gras par acidification: $5^{\circ}$ Extraction des acides gras par l'éther de pétrole 50-60.

$2^{\circ}$ Les dosages ont porté sur des tourteaux de lin et d'arachide, des grains (avoine, orge), du son de blé, des betteraves demi-sucrières, du foin de pré et de luzerne. Des échantillons de fèces de bovins ont été également étudiés.

$3^{\circ}$ L'écart relatif entre deux dosages successifs, effectués sur un même échantillon, a été trouvé généralement inférieur à $5 \%$, en ce qui concerne les acides gras.

$4^{\circ}$ La somme "glycérides + insaponifiables " a été comparée aux taux de matières grasses totales, obtenu par deux extractions successives à l'alcool puis à l'éther, dans un appareil de Soxhlet. Les deux séries de valeurs ont été sensiblement identiques pour les tourteaux. L'extraction par les solvants dans les conditions expérimentales décrites n'a pas permis de doser la totalité des lipides des grains et du son de blé. En revanche, les foins ont cédé aux solvants des substances non lipidiques (comprenant vraisemblablement de la chlorophylle).

$5^{\circ}$ La méthode de simple extraction à l'éther ou à l'éther de pétrole ne permet pas de mesurer la teneur réelle des aliments en lipides. La méthode de double extraction à l'alcool et à l'éther, bien que plus satisfaisante, ne permet pas non plus d'atteindre pleinement ce but, sauf pour les tourteaux.

$6^{0}$ Il n'existe, actuellement, aucune méthode à la fois spécifique, rapide et fidèle, qui puisse permettre de doser les acides gras des fourrages, et la seule technique possible pour effectuer ces dosages d'une manière complète se prête malheureusement assez mal aux dosages en grande série. La simplification de cette technique semble donc s'imposer.

$7^{\circ}$ Pour les raisons qui précèdent, les données des tables de composition des aliments, faisant connaître les teneurs en matières grasses brutes et digestibles, ne doivent être acceptées qu'à titre de grossière approximation.

\section{BIBLIOGRAPHIE}

(I) Association of Official Agricultural Chemists. Methods of Analysis, p. 346, 1950, Washington.

(2) Brouwer (E.). - De la composition des acides gras de l'herbe, du foin et du trèfle rouge eu égard à leur influence sur la composition de la matière grasse du beurre. Rec. Trav. chim., 62, p. 380, r943.

(3) Chibnall (A. C.), Channon (H. J.). - The ether soluble substances of cabbage leaf cytoplasm III the fatty-acids. Bioch. J., 21, p. 479, 1927. 
(4) Inaba (R.). - Uber die Feetbestimmungen der Faeces und einiger Nahrungsmittel nach der neuen Methode von Kumagawa-Suto. Bioch. Zeits., 8, p. 348, r908.

(5) Kumagawa (M.), Suto (K.). - Ein neues Verfahren zur quantitativen Bestimmung der Fettes und der unverseifbaren Substanzen in tierischem Material nebst der Kritik einiger gebrauchlichen Methoden. Bioch. Zeits., 8, p. 212, 1908.

(6) Législation des Aliments des Animaux. Éd. S.E.P., 42, rue du Louvre, Paris.

(7) Lemeland (P.) - - Dosage des acides gras totaux et de l'insaponifiable total. Bull. Soc. Chim. Biol., 3, p. 134, 192 r.

(8) Machebceuf (M.). - Etat des lipides dans la matière vivante. Hermann, Paris, I936.

(9) Maynard (L.-A.), McCay (C. M.), Loosli (J. K.), Lingensfelter (J. F.), Barrentin (B.), Spering (G.). - Physiologie de la lactation. Influence de l'huile de lin et de l'huile de coprah sur le taux de matières grasses et la qualité de la matière grasse produite. Cornell Agr. Exp. Sta. Rept., p. I00, 1942, in C. A., 39, p. 4997, I945.

( io) QueTel (R.). - Les lipides du marron d'Inde. Conditions d'extraction. Technique du dosage en série des acides gras. Bull. Soc. chim. Biol., 25, p. 224, I943.

(i I) RANDLE (S. B.). - The determination of fat in cooked animal feeds containing cereal. J. Assoc. off. Agr. chem., 26, p. 340, I943.

(i2) Schall (E. D.), Thornton (M. H.). - Methods for the determination of fat in dog food. J. Assoc. off. Agr. Chem., 26, p. 404, 1943 .

(I3) TAYLOR (J. J.). - Report on the determination of crude fat ether extract in feed mixture. J. Assoc. off. Agr. Chem., 28, p. 771, 1945.

(i4) Terroine (E. F.), Lepage (G.), Vechot (J.), Wolff (A.). - La détermination des matières grasses dans les produits végétaux. Ann. Fals., 20, p. 79, 1927.

(I5) VORIS (S. S.). - Report on the analyses of baked products other than bread. J. Assoc. off. Agr. Chem., 24, p. 624, i94r. 